\title{
Strategies to Improve Child Health
}

This is a draft of a chapter that has been accepted for publication by Oxford University Press in the book 'Oxford Textbook of Global Health of Women, Newborns, Children, and

Adolescents' edited by Delan Devakumar, Jennifer Hall, Zeshan Qureshi, and Joy Lawn, published in December 2018 (ISBN: 9780198794684).

This chapter examines the key priorities facing child health practitioners and policymakers to build on recent improvements in global child health and wellbeing. It analyses what has underpinned these successes, why shortcomings persist and how best to address them.

\section{Key points}

- Scaling up established health interventions, particularly in primary care, while addressing fundamental social determinants has substantially reduced child mortality in many countries.

- This 'success' is, however, partial and unfairly distributed. The most important child health priorities today are to:

○ understand and address between- and within-country child health inequities;

○ ensuring every child not only survives but also attains their full state of development and well-being.

- Addressing these priorities requires locally adapted strategic frameworks that coproduce child health policy with patients and communities.

\section{Background}

There is much to celebrate about recent progress in child health. Global under-five mortality has reduced by $52 \%$ over the past 25 years, with 58 countries achieving a two-thirds reduction (Wang et al., 2016). Child health improvements are broadly attributable to two approaches: scaling up key health interventions and improving underlying social determinants. These are not separate agendas - both are co-dependent and mutually reinforcing. Effectively scaling up essential child health interventions has proved capable of overcoming poverty and inequality (see also Case Study 1).

Yet the global child health agenda remains incomplete. Global reductions in child deaths conceal wide variations in individual countries' success in reducing child mortality. Many regions and countries have made worryingly little progress in addressing child health disparities, in particular Sub-Saharan Africa where Under Five mortality is still 23 times higher than in Western Europe (Wang et al., 2016). Furthermore, child health is not just about survival. Child mortality reductions now need to be matched by improvements in child development and wellbeing. 


\section{Global Strategy for Women's, Children's and Adolescent Health}

An integral component of the Sustainable Development Goals (SDGs), the Global Strategy's key objectives and child health targets (Table 1) address a number of key limitations of previous global initiatives such as the Millennium Development Goals, including:

1. Pursuing equity between countries by setting an absolute rather than relative child survival target for every country;

2. Prioritising child wellbeing and development equally with survival;

3. Explicitly recognising the interdependence of health with social, economic and political determinants through an integrated, multi-sector approach.

The Global Strategy sets out key 'action areas' and cross-cutting 'guiding principles' that will enable global child health initiatives global child health to truly 'survive, thrive and transform' across the continuum of care (Table 2).

\section{Child health across the continuum of care}

As emphasised throughout this book, child health strategies should be positioned within a lifecycle approach. Maternal nutrition, education and newborn care are particularly crucial to child health — and vice versa.

\section{Primary Health Care}

In contrast to maternal or neonatal health, most child mortality and morbidity can be effectively prevented or treated in primary care-including community outreach. Intervention packages with the most potential to save lives are:

- Infant and young child feeding especially during the first 1000 days: enabling exclusive breastfeeding for 6 months; continued breastfeeding and complementary feeding from 6 months onwards.

- Responsive caregiving and stimulation to enable carers to provide play and communication activities using household items, and to strengthen responsive care, using for example the UNICEF/WHO Care for Child Development package.

- Immunisation through universal coverage of the Extended Programme for Immunisations, plus periodic vitamin A supplementation where appropriate.

- Integrated management of acute illnesses: using Integrated Management of Newborn and Childhood Illnesses (IMNCI) and Integrated Community Case Management of Childhood Illness (iCCM) approaches to target pneumonia, diarrhoea, sepsis, malnutrition and (where endemic) malaria in particular.

There is clear, established evidence for affordable community and clinical interventions in each of these areas, as outlined in Chapters 36 and 37. Where these interventions have been effectively rolled out at scale, child mortality has consistently dropped. Yet coverage remains incomplete, particularly amongst the most vulnerable and marginalised populations (Figure 1). For example, child survival rates in the poorest economic quintile are half that of the richest.

The evidence-base supporting child development and wellbeing interventions in resource-poor settings emphasises the importance of responsive and nurturing care, the treatment and rehabilitation of disabilities, maternal mental health and adequate social protection to reduce and protect against poverty. 
The strategic priority today is to understand and address the reasons for implementation gaps. In particular, child health initiatives need to engage better with local contexts by not just asking 'what works' clinically but 'what works here' programmatically, through implementation and social science research into local situational understandings of health, social and political systems.

\section{Hospital Care}

Primary care services cannot function in isolation. Mutually supportive links with both inpatient services and communities are needed to deliver comprehensive, quality and trusted primary care services. Children whose illnesses or injuries are not manageable in primary care - malaria or severe acute malnutrition with complications, or road traffic accidents for example - need timely referral to secondary care.

Even in secondary care, clinical care priorities should focus on the quality delivery of relatively simple interventions to manage severe illnesses and injuries, especially in the first 24 hours of admission. Prompt emergency assessment and treatment by staff trained and equipped to safely provide intravenous fluids, antibiotics and blood transfusions save more lives than hightechnology intensive care equipment. Again, service fragmentation should be avoided by strengthening hospital care using an integrated approach. In addition, as countries go through the epidemiological transmission, increasing numbers of children will suffer from more complex chronic conditions that are better managed at higher-level facilities.

Functional links between primary and secondary care are also important for health staff. Effective referral pathways avoid frustration with limited local capacity and reduce time spent away from other essential primary health activities. Such links also strengthen supportive supervision and continuing professional education that build staff motivation and quality.

\section{Community and intersectoral priorities}

Strong, equitable governance mechanisms between health workers and the communities they serve are crucial to fostering a responsive and accessible health system. Communities are also central to addressing wider social, economic and political health determinants, maintaining the economic and social stability that is fundamental to child health and wellbeing.

Key child health determinants include:

- local and planetary environments that are conducive to child health and development, in particular access to water, sanitation and hygiene;

- sustainable livelihoods;

- food security and nutrition, including breast feeding;

- education, both as a direct component of child wellbeing and for children as future parents and healthworkers.

Local action at household and community level is important in addressing these broader child health priorities. Such initiatives will only succeed, however, within enabling economic and political environments created by fundamental intersectoral transformations at national and global levels. 


\section{Child health systems}

The most important health system weaknesses limiting equitable access to child health services are: financial barriers to care; shortages or mismatched distributions of trained health staff and essential medicines; and governance structures that are unresponsive to local needs (see also Case study 2 and Chapter 8).

Increasing geographical coverage of quality child health services and removing user fees are both key to improving access and protecting families from catastrophic health costs. Where child health strategies do not reflect local concerns, health intervention uptake suffers. Child health strategies must therefore include explicit mechanisms that enable local adaptation of child health initiatives to address both local and global priorities. Locally responsive child health services also require health systems that are 'people-centred' and 'community-owned', where control over priority-setting is shared and negotiated within collaborative partnerships between policymakers, health practitioners and citizens.

\section{Controversies/Challenges}

How can we co-produce child health initiatives with families and communities? Barriers include socio-cultural differences between health workers and their patients, hierarchical health management structures that restrict local adaptation, and governments with little incentive to change the societal power dynamics that brought them to power. There are, however, increasing examples of countries successfully overcoming these barriers (see for example Case Study 1).

Technical changes tend to be more readily achieved than social and political transformations. For example, the Integrated Management of Childhood Illness became largely synonymous with improved clinical case management rather than the wider transformation of health and social systems that was originally intended. We need to ensure that the 'thrive' and 'transform' child health agendas are not eclipsed by 'survive' alone.

\section{Questions}

1. How does the Global Strategy differ from previous global child health initiatives?

2. What are the key differences in child health priorities currently facing Sierra Leone and Ethiopia?

\section{Conclusions}

A holistic and multi-sectoral strategy targeting primary, secondary and community levels of care as well as broader social determinants is central to improving child health and wellbeing. Child health strategies must actively engage with the complexity and context-dependence of health interventions when rolled out at scale. This is best achieved by more equitably balanced decision-making relationships between health professionals and citizens that champion local situational knowledge and priorities equally with global evidence.

\section{Case study 1 - Ethiopia}

Between 1990 and 2009, after two decades of severe political and economic instability and despite persistently poor economic growth, Ethiopia halved under-five mortality across all its ethnically and socio-economically diverse regions. Major reductions were achieved in malaria 
mortality, undernutrition, and the numbers of families with poor access to water and sanitation. The 2003 Health Extension Program played a particularly important role in scaling up key health interventions delivered by large numbers of health extension workers.

Key factors underpinning these successes include:

- coherent policy approaches across health priorities, contributing to 'horizontal' improvements in health system strengthening and household gender equality;

- explicitly including health in poverty reduction and sustainable development policies;

- Adopting a health system governance model enabling local needs to be prioritised within national targets.

\section{Case study 2 - Sierra Leone}

In 2002, after a protracted civil war preceded by a major economic recession, child health in Sierra Leone was among the worst in the world. A decade of investment in the health sector, notably with the 2010 Free Health Care Initiative, had some success with reducing child mortality - albeit more slowly than hoped. The fragility of this progress, however, was tragically shown in 2014 with the outbreak of an unprecedented Ebola epidemic.

Sierra Leone's experience and efforts to rebuild a more 'resilient' health system holds important lessons for addressing child health globally:

- Health policy should be designed for when things go wrong as well as when things go right. Like many countries with high childhood illness, Sierra Leone's health system is characterised by crisis more often than stability.

- Actively building trust between citizens and healthworkers is crucial not only for achieving long-term child health goals, but for withstanding and recovering from acute crises.

Table 1: adapted from Every Woman Every Child (2015)

\section{Global Strategy: Objectives and key child health targets}

Survive - end preventable deaths

- Reduce under-five mortality to below 25 per 1,000 live births in every country.

Thrive - ensure health and wellbeing

- End all forms of malnutrition and address the nutritional needs of children.

- Ensure all girls and boys have access to good quality early childhood development.

- Achieve universal health coverage, including financial risk protection and access to quality essential services, medicines and vaccines.

\section{Transform - expand enabling environments}

- Eradicate extreme poverty.

- Ensure all girls and boys complete free, equitable and good-quality primary and secondary education.

- Eliminate all harmful practices, discrimination and violence against women and girls.

- Achieve universal and equitable access to safe drinking water, sanitation and hygiene. 
Table 2: Every Woman Every Child (2015)

\section{Global Strategy: Action Areas}

- Country leadership

- Financing for health

- Health system resilience

- Individual potential

- Community engagement

- Multi-sector action

- Humanitarian and fragile settings

- Research and Innovation

- Accountability

\section{Global Strategy: Guiding Principles}

Country-led, Universal, sustainable, rights-based, gender-responsive, evidence-informed, partnership-driven, people-centred, community-owned, accountable and aligned with development effectiveness and humanitarian norms.

Figure 1 - Under-fives deaths by economic and maternal education status. Adapted from Every Women Every Child (2015:30)

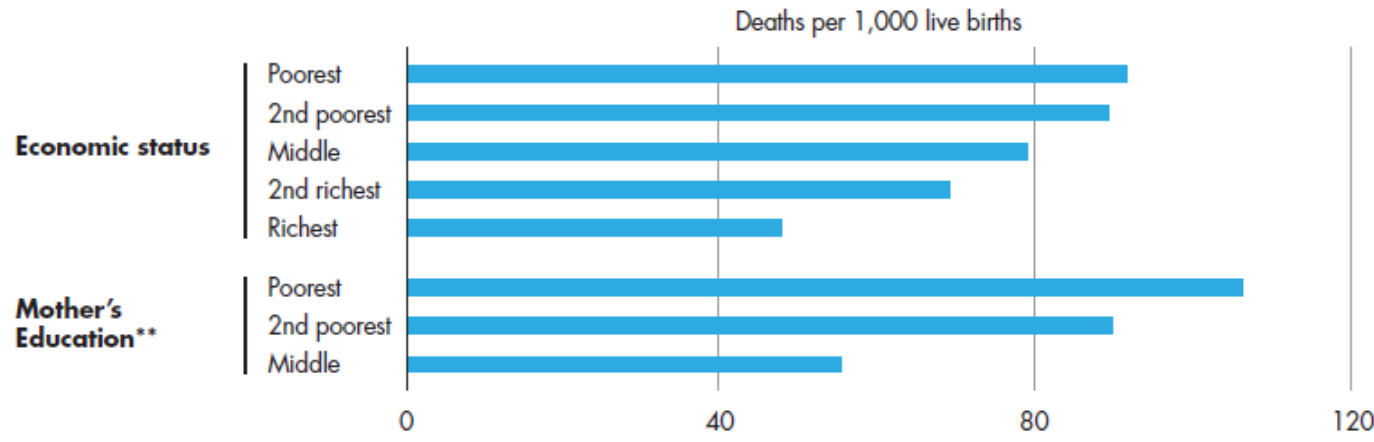

* Data from national Demographic and Health Surveys in 49 low-and middle-income countries, 2005-2012. ** Education data are not available for 10 countries. $^{39}$

\section{Key publications}

Past challenges and future priorities in child health strategies:

BRYCE J et al. 2013. The unfinished agenda in child survival. Lancet, 382, 1049-1059.

Full details of the Global Strategy:

EVERY WOMAN EVERY CHILD 2015. The Global Strategy For Women's, Children's and Adolescents' Health (2016-2030). Italy: Every Woman Every Child.

In-depth analysis of community participation, including key implementation challenges:

MARSTON C et al. 2016. Community participation for transformative action on women's, children's and adolescents' health. Bulletin of the World Health Organization, 94, 376-382. 


\section{Full Bibliography}

BALABANOVA, D., MCKEE, M. \& MILLS, A. 2011. 'Good Health At Low Cost' 25 Years On. What makes a successful health system?, London, London School of Hygiene and Tropical Medicine.

BANTEYERGA, H., KIDANU, A., CONTEH, L. \& MCKEE, M. 2011. Ethiopia - Placing health at the centre of development. Chapter 4. In: BALABANOVA, D., MCKEE, M. \& MILLS, A. (eds.) 'Good Health At Low Cost' 25 Years On. What makes a successful health system? London: London School of Hygiene and Tropical Medicine.

BRYCE, J., VICTORA, C. G. \& BLACK, R. E. 2013. The unfinished agenda in child survival. The Lancet, 382, 1049-1059.

DAELMANS, B., BLACK, M. M., LOMBARDI, J., LUCAS, J., RICHTER, L., SILVER, K., ... RAO, N. 2015. Effective interventions and strategies for improving early child development. $B M J, 351,23-26$.

EVERY WOMAN EVERY CHILD 2015. The Global Strategy For Women's, Children's and Adolescents' Health (2016-2030). Italy: Every Woman Every Child.

GOVERNMENT OF SIERRA LEONE 2015. National Ebola Recovery Strategy For Sierra Leone 2015-2017. Freetown: Government of Sierra Leone.

LARSON, H. \& SCHULZ, W. 2015. The State of Vaccine Confidence 2015. London: The Vaccine Confidence Project, London School of Hygiene and Tropical Medicine.

MARSTON, C., HINTON, R., KEAN, S., BARAL, S., AHUJA, A., COSTEllo, A. \& PORTELA, A. 2016. Community participation for transformative action on women's, children's and adolescents' health. Bulletin of the World Health Organization, 94, 376382.

SHAW, B., AMOUZOU, A., MILLER, N. P., TAFESSE, M., BRYCE, J. \& SURKAN, P. J. 2016. Access to integrated community case management of childhood illnesses services in rural Ethiopia: a qualitative study of the perspectives and experiences of caregivers. Health Policy and Planning, 31, 656-666.

SHEIKH, K., RANSON, M. K. \& GILSON, L. 2014. Explorations on people centredness in health systems. Health Policy and Planning, 29 iil-ii5.

UNICEF/WORLD HEALTH ORGANIZATION 2012. Care for Child Development Package. Geneva: World Health Organization.

WANG, H., BhUTTA, Z. A., COATES, M. M., COGGEShall, M., DANDONA, L., DIALLO, K., . . . MURRAY, C. J. L. 2016. Global, regional, national, and selected subnational levels of stillbirths, neonatal, infant, and under-5 mortality, 19802013;2015: a systematic analysis for the Global Burden of Disease Study 2015. The Lancet, 388, 1725-1774.

WATKINS, K. 2016. Longer lives and unfinished agendas on child survival. The Lancet, 388, $1450-1452$.

WITTER, S., BRIKCI, N., HARRIS, T., WILlIAMS, R., KEEN, S., MUJICA, A., . . . RENNER, A. 2016. The Sierra Leone Free Health Care Initiative (FHCI): process and effectiveness review. Final Report. Oxford: Oxford Policy Management.

WORLD HEALTH ORGANIZATION 2016. Towards a grand convergence for child survival and health: a strategic review of options for the future building on lessons learnt from IMNCI. Geneva: WHO. 